\title{
A MEAN OSCILLATION INEQUALITY
}

\author{
IVO KLEMES
}

\begin{abstract}
It is proved that $\left\|f^{*}\right\|_{\mathrm{BMO}} \leqslant\|f\|_{\mathrm{BMO}}$, where $f^{*}$ is the decreasing rearrangement of a function $f \in \operatorname{BMO}([0,1])$. A generalization is given, as well as an example, showing the result fails for the symmetric decreasing rearrangement of a function on the circle.
\end{abstract}

In this paper we prove that

\section{(*)}

$\|f *\|_{\mathrm{BMO}} \leqslant\|f\|_{\mathrm{BMO}}$

where $f^{*}$ is the decreasing rearrangement of the function $f:[0,1] \rightarrow \mathbf{R}$. The sharp constant one in this inequality refines a result contained in Theorem 3.1 of [1]. We also indicate generalizations, and finally mention symmetric decreasing rearrangement on the circle, for which (*) fails.

The average of $f$ over $E \subset[0,1],|E|>0$, will be denoted by $f_{E}=(1 /|E|) \int_{E} f$. The definition of the norm in $(*)$ is

$$
\|f\|_{\mathrm{BMO}}=\sup _{J \subset[0,1]} \frac{1}{|J|} \int_{J}\left|f-f_{J}\right|,
$$

where $J$ ranges over all subintervals of $[0,1]$. We need the following version of the rising sun lemma. See [3] or [2, p. 293] for the usual rising sun lemma.

LemMa. Let $f \in L^{1}([0,1])$ and suppose the average $f_{[0,1]} \leqslant \alpha$. Then there is a finite or countable set $\mathscr{L}$ of pairwise disjoint subintervals of $[0,1]$ such that $f_{L}=\alpha$ for each $L \in \mathscr{L}$, and $f \leqslant \alpha$ almost everywhere on $[0,1] \backslash \cup \mathscr{L}$.

Proof. It suffices to prove the case $\alpha=0$. Define $F(x)=\int_{0}^{x} f$ on $[0,1]$. Choose $t_{0} \geqslant 0$ as large as possible so that $F\left(t_{0}\right)=0$. Let $\mathcal{O}=\left\{t \in\left[t_{0}, 1\right]: F(x)>F(t)\right.$ for some $x>t\}$. Then $\mathcal{O}$ is open in $\left[t_{0}, 1\right]$, so $\mathcal{O}=\bigcup I_{j}$ for some disjoint intervals $I_{j}$ open in $\left[t_{0}, 1\right]$. Defining $\mathscr{L}$ to be these intervals together with $\left[0, t_{0}\right)$ satisfies the lemma, as we now verify. On $\left[0, t_{0}\right)$ the average of $f$ is 0 by definition. Let $I_{j}$ have endpoints $t_{0} \leqslant a_{j}<b_{j} \leqslant 1$.

If $F\left(b_{j}\right)>F\left(a_{j}\right)$ then $a_{j} \in \mathcal{O}$ by definition, so $a_{j}=t_{0}$. But, by hypothesis, $F(1)=f_{[0,1]} \leqslant 0=F\left(t_{0}\right)=F\left(a_{j}\right)<F\left(b_{j}\right)$, so (by the intermediate value theorem) $F(c)=0$ for some $b_{j}<c \leqslant 1$, contradicting the choice of $t_{0}$.

If $F\left(b_{j}\right)<F\left(a_{j}\right)$ choose $\tau \in\left[a_{j}, b_{j}\right]$ as large as possible so that

$$
F(\tau)=\frac{1}{2}\left(F\left(a_{j}\right)+F\left(b_{j}\right)\right)
$$

Received by the editors March 25, 1984 and, in revised form, June 27, 1984 1980 Mathematics Subject Classification. Primary 26A87. 
Then $a_{j}<\tau<b_{j}$, so $\tau \in \mathcal{O}$, and, by definition, $F(x)>F(\tau)$ for some $x>\tau$. In fact, $x>b_{j}$ by the choice of $\tau$, hence $b_{j} \in \mathcal{O}$ and $b_{j}<1$. But then $b_{j}$ is not an endpoint of $I_{j}$, contradiction.

We conclude $F\left(b_{j}\right)=F\left(a_{j}\right)$, whence the average $f_{I_{j}}=0$, as desired. Finally, by the Lebesgue differentiation theorem, $f(t)=\lim _{x \downarrow t}(F(x)-F(t)) /(x-t)$ for almost all $t \in[0,1]$. Since $F(x) \leqslant F(t)$ when $x>t \in\left[t_{0}, 1\right] \backslash \mathcal{O}$, we conclude $f(t) \leqslant 0$ almost everywhere on $[0,1] \backslash \cup \mathscr{L}$.

By visualizing the above proof the reader may notice that it had more to do with water levels than sun rays.

Proof of (*). Fix an interval $J \subset[0,1]$ and define $\alpha=f_{J}^{*}$. We assume $f_{[0,1]} \leqslant \alpha$ since an entirely symmetrical argument will cover the case $f_{[0,1]} \geqslant \alpha$. Apply the lemma to $f$ and $\alpha$ and set $E=\bigcup \mathscr{L}$. To prove (*) we have but to show

$$
\frac{1}{|J|} \int_{J}\left|f^{*}-\alpha\right| \leqslant \frac{1}{|E|} \int_{E}|f-\alpha|
$$

because then we may finish as follows:

$$
\frac{1}{|E|} \int_{E}|f-\alpha|=\frac{\sum_{L \in \mathscr{L} \int_{L}}|f-\alpha|}{\sum_{L \in \mathscr{L}}|L|} \leqslant \sup _{L \in \mathscr{L}} \frac{1}{|L|} \int_{L}|f-\alpha| \leqslant\|f\|_{\mathrm{BMO}}
$$

Now $(* *)$ can be proved in two steps.

First choose $t$ as large as possible so that $(1 / t) \int_{0}^{t} f^{*}=\alpha$. $t$ exists because $f_{[0,1]}^{*} \leqslant \alpha$ and $f_{J}^{*}=\alpha$. We claim that

$$
\frac{1}{|J|} \int_{J}\left|f^{*}-\alpha\right| \leqslant \frac{1}{t} \int_{0}^{t}\left|f^{*}-\alpha\right|
$$

To see this, choose a point $\tau \in J$ such that $f^{*}(x) \geqslant \alpha$ for $x<\tau$ and $f^{*}(x) \leqslant \alpha$ for $x>\tau$. Let $J=\left[\tau-x_{1}, \tau+x_{2}\right]$ and $[0, t]=\left[\tau-y_{1}, \tau+y_{2}\right]$. Define

$$
A \equiv \int_{\tau-x_{1}}^{\tau}\left|f^{*}-\alpha\right|=\int_{\tau}^{\tau+x_{2}}\left|f^{*}-\alpha\right|
$$

and

$$
B \equiv \int_{0}^{\tau}\left|f^{*}-\alpha\right|=\int_{\tau}^{t}\left|f^{*}-\alpha\right| .
$$

The monotonicity of $\left|f^{*}-\alpha\right|$ on $[0, \tau)$ implies $A / x_{1} \leqslant B / y_{1}$. Similarly on $(\tau, t]$ we get $A / x_{2} \leqslant B / y_{2}$. Taking reciprocals and adding (in the nontrivial cases) we get $\left(x_{1}+x_{2}\right) / A \geqslant\left(y_{1}+y_{2}\right) / B$ or $2 A /\left(x_{1}+x_{2}\right) \leqslant 2 B /\left(y_{1}+y_{2}\right)$, which is (1).

The second step is to prove

$$
\frac{1}{t} \int_{0}^{t}\left|f^{*}-\alpha\right| \leqslant \frac{1}{|E|} \int_{E}|f-\alpha| .
$$

In fact, we will show $t \geqslant|E|$ and $\int_{0}^{t}\left|f^{*}-\alpha\right|=\int_{E}|f-\alpha|$.

By properties of $f^{*}$, the identity $\int_{0}^{|E|} f^{*} \geqslant \int_{E} f$ holds for any set $E$. For our $E$ it implies

$$
\frac{1}{|E|} \int_{0}^{|E|} f^{*} \geqslant \frac{1}{|E|} \int_{E} f=\alpha,
$$

whence $t \geqslant|E|$ by the definition of $t$ and monotonicity of $f^{*}$. 
Finally, recall that $f \leqslant \alpha$ almost everywhere on $[0,1] \backslash E$. Hence, we can argue

$$
\begin{aligned}
\int_{0}^{t}\left|f^{*}-\alpha\right| & =2 \int_{\left\{x: f^{*}(x)>\alpha\right\}} f^{*}-\alpha=2 \int_{\{x: f(x)>\alpha\}} f-\alpha \\
& =2 \int_{\{x: f(x)>\alpha\} \cap E} f-\alpha=\int_{E}|f-\alpha| .
\end{aligned}
$$

This concludes the proof of $(*)$.

The existence of the intermediate quantity $(1 / t) \int_{0}^{t}\left|f^{*}-\alpha\right|$ (the "worst case" for $f^{*}$ ) and the apparently distinct reasons for the inequalities on either side of it struck the author as rather arresting. Furthermore, there appeared to be lurking a curious symmetry between the behaviour of the numerators and denominators in the whole proof. Both of these points were happily resolved by

THEOREM. Let $f \in L^{1}([0,1])$. Suppose $J \subset[0,1]$ is an interval and $E \subset[0,1]$ is a set such that

(i) $f_{\cdot J}^{*}=f_{E} \equiv \alpha$ and

(ii) $\int_{J}\left|f^{*}-\alpha\right| \leqslant \int_{E}|f-\alpha|$.

Suppose further that $F, G:[0, \infty) \rightarrow[0, \infty)$ are such that $F(\lambda) / \lambda$ is increasing and $G(\lambda) / \lambda$ is decreasing for $\lambda>0, F(0)=0$, and $F \circ|f-\alpha|, G \circ|f-\alpha| \in L^{1}([0,1])$. Then

$$
\frac{\int_{J} F \circ\left|f^{*}-\alpha\right|}{\int_{J} G \circ\left|f^{*}-\alpha\right|} \leqslant \frac{\int_{E} F \circ|f-\alpha|}{\int_{E} G \circ|f-\alpha|}
$$

(where we take $0 / 0=0$ and $x / 0=\infty, x>0$ ).

The author has a proof which involves writing the integrals in terms of distribution functions of the kind $m(\lambda)=\int_{\{t: f(t)-\alpha>\lambda\} \cap E} f-\alpha$ and using integration by parts. We can choose $F(\lambda)=\lambda^{p}, 1 \leqslant p<\infty, G(\lambda)=1$ and, using the same set $E$ as in our earlier argument, obtain $(*)$ with exponent $p$ in place of 1 .

We now consider functions of bounded mean oscillation on the circle $T \equiv \mathbf{R} / 2 \pi \mathbf{Z}$. For $f \in \operatorname{BMO}(T)$ define

$$
\|f\|=\sup _{J} \frac{1}{|J|} \int_{J}\left|f-f_{J}\right|,
$$

where $J$ ranges over all intervals in $T$. Let $f^{\#}$ denote the symmetric decreasing rearrangement of $f$. Making use of $(*)$, it can be shown that $\left\|f^{\#}\right\| \leqslant 2\|f\|$. However, there are functions $f$ such that $\left\|f^{\#}\right\|>\|f\|$. We give an example, omitting the computations.

Define $f(0)=f(1)=f(-1)=0, f\left(\frac{1}{3}\right)=f\left(-\frac{1}{3}\right)=1$ and interpolate linearly for the remaining $\theta \in[-\pi, \pi]$. Then $f^{\#}$ is the piecewise linear function with corners $(-1,0)$, $(0,1),(1,0)$, and it can be shown that $\left\|f^{\#}\right\|>\|f\|$.

The failure of the inequality $\left\|f^{\#}\right\| \leqslant\|f\|$ is due to the fact that the supremum $\left\|f^{\#}\right\|$ may be achieved for an interval $J$ on which $f^{\#}$ is not monotone. This makes it possible to construct an (equimeasurable) "perturbation" $f$ for which $\|f\|<\left\|f^{\#}\right\|$ (as in the above example). 
We conclude with two problems:

(1) For $f \in \operatorname{BMO}(T)$ are there any equimeasurable rearrangements $g$ for which $\|g\|$ is minimal? If so, describe them.

(2) What is the best constant $c$ such that $\left\|f^{\#}\right\| \leqslant c\|f\|$ for all $f \in \operatorname{BMO}(T)$ ?

ACKNowledgements. I want to thank Professor T. H. Wolff for suggesting the problems in this paper and pointing out the relevance of the rising sun lemma. The basic covering strategy was motivated by the proof of Theorem 3.1 in [1]. I also want to acknowledge the referee's suggestion to concentrate the presentation on the case $p=1$.

\section{REFERENCES}

1. C. Bennett, R. A. DeVore, and R. Sharpley, Weak- $L^{\infty}$ and BMO, Ann. of Math. (2) 113 (1981), 601-611.

2. G. H. Hardy, J. E. Littlewood, and G. Pólya, Inequalities, Cambridge Univ. Press, 1967.

3. F. Riesz, Sur un théorème de maximum de Mm. Hardy et Littlewood, London Math. Soc. 7 (1932), $10-13$.

Department of Mathematics, California Institute of Technology, Pasadena, California 91125 